\title{
The X-Ray Surveyor Mission Concept Study: Forging the Path to NASA Astrophysics 2020 Decadal Survey Prioritization \\ Jessica Gaskin $^{1 \mathrm{a}}$, Feryal Özel ${ }^{\mathrm{b}}$, Alexey Vikhlinin ${ }^{\mathrm{c}}$ \\ On behalf of the X-Ray Surveyor STDT
}

\author{
${ }^{a}$ NASA Marshall Space Flight Center, Huntsville, AL USA 35812; ' Department of Astronomy, \\ University of Arizona, 933 N. Cherry Ave., Tucson, AZ USA 85721, 'Smithsonian Astrophysical \\ Observatory, 60 Garden Street, Cambridge, MA 02138
}

\begin{abstract}
The X-Ray Surveyor mission concept is unique among those being studied for prioritization in the NASA Astrophysics 2020 Decadal Survey. The X-Ray Surveyor mission will explore the high-energy Universe; providing essential and complimentary observations to the Astronomy Community. The NASA Astrophysics Roadmap (Enduring Quests, Daring Visions) describes the need for an X-Ray Observatory that is capable of addressing topics such as the origin and growth of the first supermassive black holes, galaxy evolution and growth of the cosmic structure, and the origin and evolution of the stars that make up our Universe. To address these scientifically compelling topics and more, an Observatory that exhibits leaps in capability over that of previous X-Ray Observatories in needed. This paper describes the current status of the X-Ray Surveyor Mission Concept Study and the path forward, which includes scientific investigations, technology development, and community participation.
\end{abstract}

Keywords: X-Ray Surveyor, X-Ray Astronomy, NASA Astrophysics Mission Concept Study

\section{INTRODUCTION}

\subsection{X-Ray Surveyor Mission Concept Study}

The opportunity to conceive of, design, and fly a truly Great Observatory only comes around once a decade. Previous decades have hosted successes such as the Hubble Space Telescope and the Chandra X-Ray Observatory (CXO). To be launched this decade and the next are the James Webb Space Telescope (JWST) and Wide Field Infrared Survey Telescope (WFIRST), respectively. The 2020 Astrophysics Decadal Review Committee will be tasked to consider and prioritize four large mission concepts brought forth by the Astronomy Community. The $X$ Ray Surveyor is one of these, and is the only mission of the four that will explore the high-energy Universe.

Supporting the Astrophysics Decadal Review Committee in their prioritization of these four large missions requires providing them with a study package that includes a fully-defined science case, a design reference mission, a technology roadmap, and a rough order of magnitude cost and schedule. Study products for each of the four missions are produced under the direction of NASA HQ-appointed Science and Technology Definition Teams (STDT). The STDTs consist of members from the Community. Each STDT is supported by a Study Office located at a NASA Center. X-Ray Surveyor is the exception to this, as the Study Office is a partnership between NASA Marshall Space Flight Center (MSFC) and the Smithsonian Astronomical Observatory (SAO). The MSFC-SAO partnership has a long and successful history regarding X-ray mission development and flight that started over 40 years ago and has continued through the successful launch and operation of the $\mathrm{CXO}^{1}$.

\subsection{The X-Ray Surveyor Study Team}

The X-Ray Surveyor Study Team consists of the STDT, the MSFC-SAO Study Office, non-voting Observers and International representatives.

\footnotetext{
${ }^{1}$ Jessica.Gaskin@nasa.gov; phone 1256 961-7818; http://wwwastro.msfc.nasa.gov/xrs/
} 
The X-Ray Surveyor STDT is a diverse team with members from across the community, and whose backgrounds range from early universe to large scale structures, to stellar astronomy. This team is responsible for the generation and delivery of all study products to the Astrophysics Decadal Review Committee. STDT Co-Chairs are Feryal Özel (U Arizona) and Alexey Vikhlinin (SAO). Team members include: Steve Allen (Stanford), Mark Bautz (MIT), Niel Brandt (Penn State), Joel Bregman (Michigan), Megan Donahue (MSU), Jessica Gaskin (MSFC), Ryan Hickox (Dartmouth), Tesla Jeltema (UCSC), Juna Kollmeier (OCIW), Laura Lopez (Ohio State), Piero Madau (UCSC), Rachel Osten (STScI), Frits Paerels (Columbia), Mike Pivovaroff (LLNL), Dave Pooley (Trinity), Andy Ptak (GSFC), Eliot Quataert (Berkeley), Chris Reynolds (UMD), Daniel Stern (JPL).

The MSFC-SAO Study Office provides support for all aspects of the STDT concept development. Members at MSFC include: Jessica Gaskin (NASA-Appointed Study Scientist), Douglas Swartz (Deputy Study Scientist), Martin Weisskopf (Senior Advisor) and Mark King (Study Manager). At SAO, members include: Alexey Vikhlinin (Science and Technology Advisor), Harvey Tananbaum (Senior Advisor) and Lester Cohen (Chief Telescope Engineer for the Study Office).

Observers and International Representatives play a critical role in this Study. Their guidance is essential to maintaining the Big Picture and identifying potential support on a global scale. Observers and International Representatives for $X$ Ray Surveyor include: Daniel Evans (Program Manager, NASA HQ), Ann Hornschemeier (Physics of the Cosmos Chief Scientist, NASA HQ), Robert Petre (General Advisor, GSFC), Randall Smith (Athena Liaison, SAO), Kirpal Nandra (German Aerospace Center, DLR Appointed), Brian McNamara (Canadian Space Agency, CSA Appointed), Gabriel Pratt (French Government Space Agency, CNES Appointed), Makoto Tashiro (Japan Aerospace Exploration Agency, JAXA Appointed), Arvind Parmar (European Space Agency, ESA Appointed) and Giovanni Pareschi (Italian Space Agency, ASI Appointed).

\subsection{Study Methodology and Working Groups}

Mission concept development starts with defining the science goals and then designing a feasible configuration that will meet these goals. Identification of these science goals are on-going and require community participation. To support this effort, Science Working Groups (SWGs) have been organized. These SWGs are grouped as:

- Cycles of Baryons In and Out of Galaxies

- First Accretion Light

- Evolution of Structure and AGN Populations

- Physics of Plasmas

- Physics of High Density Matter, Compact Objects, and Accretion

- Physics of Feedback

- Stellar Lifecycles

- X-Rays in the Multi-Wavelength, Multi-Messenger Era

Participation in these SWGs is open to the astronomy community. The structure of the SWGs may change as the science case evolves over the course of the Mission Study.

Understanding what a 'feasible' configuration might look like requires a complete understanding of existing technology developments for the optics and for any potential instrument suite. Similar to the Science WGs, the STDT has formed an Optics WG (OWG) and an Instrument WG (IWG). The OWG and IWG will operate in a highly collaborative manner and will work with the STDT and SWGs to develop payload requirements that are consistent as possible to the science requirements. Similar to the SWGs, the OWG and the IWG are open to the community. All of the WGs are chaired by at least one STDT member. 


\section{X-RAY SURVEYOR SCIENCE}

In this short paper, we identify a broad (and very incomplete) range of potential science topics where X-ray Surveyor might provide unique information through its sensitivity, angular resolution, and spectroscopic capabilities. As mentioned in the previous section, the STDT and SWGs have been tasked with fully identifying the science case that will be reported to the 2020 Astrophysics Decadal Review Committee.

\subsection{Understanding the Universe}

It is a great challenge to understand the complexities of the universe we live in. NASA Astrophysics is driven by three defining questions: How did we get here? How does the universe work? Are we alone? A multi-wavelength approach is required to address these questions, and astronomers have demonstrated that X-ray observations with Chandra and other missions provide an essential element to this quest. The observation of X-rays provide unique insights into the evolution of the universe from early epochs to the present while probing and elucidating underlying physical processes on scales from cosmological to atmospheres of nearby stars and planetary systems.

Comprehensive community reports such as the 2010 Decadal Survey ${ }^{1}$ - "New Worlds, New Horizons" — and the 2013 NASA Astrophysics Roadmap ${ }^{2}$ — "Enduring Quests, Daring Visions" — summarize current knowledge and understanding while laying out many of the key questions for the decades ahead. The X-ray Surveyor is conceptualized to provide the X-ray channel(s) required along with major space and ground-based facilities such as JWST, ALMA, and $30 \mathrm{~m}$-class optical telescopes to address many of the most challenging and difficult of these questions, while greatly expanding the discovery space.

$X$-ray Surveyor is extremely well poised to uniquely contribute to fundamental studies of the roles which central black holes play in the evolution of galaxies, and shed light onto their origin. While JWST will see the earliest galaxies at redshifts 10 and beyond, it is not geared to detect the early black holes in these "first" galaxies. While observations already show that by redshifts 6-7 some supermassive black holes in quasars have grown to $\sim 10^{9} \mathrm{M}_{\text {Sun }}$ or more, very little is known about the nature of initial black hole seeds, their growth at early times, and how this growth shapes the early evolution of galaxies. Black holes with $\mathrm{M}<10^{6} \mathrm{M}_{\text {Sun }}$ are best observed in the X-ray band due to the shifting of the spectral peak to wavelengths shortward of UV, due to dust obscuration of optical and UV radiation, and due to shifting of the IR signatures of AGNs out of the JWST band for z 10 objects. X-ray Surveyor can be designed to have sufficient sensitivity to detect black holes as small as $10^{4} \mathrm{M}_{\text {Sun }}$ out to $\mathrm{z} \sim 10$ (for Eddington limited accretion and assuming $10 \%$ of the bolometric luminosity is emitted in the hard X-ray band. If more massive seeds form or grow at still earlier epochs, $X$-ray Surveyor will detect them out to redshifts 15 or greater. While JWST will detect the first galaxies, X-ray Surveyor will detect the first accretion light from central black holes in these galaxies and shed light onto their early co-evolution.

In many astrophysical settings, gas with temperatures of a million degrees or higher is an essential and at times is the dominant form of the baryonic matter, tracing previous and ongoing physical processes. Theoretical modeling predicts that, in Milky Way-sized galaxies, baryons are divided into 3 roughly comparable components - stars and cold gas within the optical radius, warm gas, and very large, pressure-supported, halos of hot ( $\mathrm{T} \sim 10^{6} \mathrm{~K}$ and higher) gas. This last component plays a crucial role in regulating galaxy formation, and preserves the record of energy feedback from black holes and other sources. Its properties are a key to understanding the evolution of galaxies and stars across cosmic time. Given that this hot gas is best observed in the X-rays, X-ray Surveyor will be able to detect and characterize these hot halos, providing the most direct observational constraint on the energy feedback. This will be a key ingredient for the development of a consistent description of galaxy formation from initial conditions to early galaxies and to the beautiful spirals and ellipticals seen today. Woven through this entire fabric is the cosmic web of dark matter and baryons feeding gas into clusters, groups, and galaxies as they form and grow and also potentially collecting matter lost from these systems. X-ray Surveyor will be able to map hot baryons beyond the halos of individual galaxies, reaching into the cosmic web filaments. High angular resolution is essential for study of the relevant physical scales. Furthermore, for some of the most demanding applications, the very detection of hot diffuse gas depends on our ability to isolate the emission from discrete point-like sources. X-ray Surveyor will provide the capability to expose hot gas with phase space parameters and at distances substantially beyond the reach of any existing or planned facilities.

In today's Universe, most of the baryons appear to be outside the virial radii of any gravitationally bound structures. Some of these baryons are warm and can be detected in the $\mathrm{O}$ and $\mathrm{Mg}$ absorption lines in the UV, but a large fraction is 
hot $\left(\mathrm{T}>10^{6} \mathrm{~K}\right)$ and can be detected via absorption features they imprint on X-ray spectra of background AGN or through their X-ray emission. X-ray Surveyor will have the angular resolution to eliminate contamination from background and foreground sources and the sensitivity to enable detection in emission of at least half of the hot, diffuse baryons confined in the filamentary structures with density contrast $\rho /$ mmean $>30$.

Additional science drivers include detailed studies of AGN feedback in clusters, groups, and galaxies; tracing winds and jets while deciphering the overall gas flow picture around supermassive black holes; unraveling the processes by which AGN jets are powered, collimated, and re-accelerated; unfolding the interactions of supernova blasts, shocks, and ejecta with circumstellar and interstellar material as well as mapping particle acceleration in supernova remnants and pulsar wind nebulae; and probing coronal activity in stars of all ages along with extending our understanding of star-planet interactions.

\subsection{Community Involvement}

One of the primary goals of the STDT is to ensure that the huge leaps in capabilities provided by the X-ray Surveyor can be exploited to address a broad range of fundamental science questions across astronomy. Owing to the intrinsically interdisciplinary nature of these science questions, we expect that the X-ray Surveyor will engage and benefit multiple astronomy communities. With this goal in mind, the STDT has sought and will continue to seek wide community input in defining and refining the $X$-ray Surveyor science goals and capabilities. One key consideration is the complementarity of the X-ray Surveyor with other major space- and ground-based astronomical facilities expected in the next decade and beyond.

The membership in the Science Working Groups of the X-ray Surveyor is open to the wide community. To further develop the breakthrough science that can be done with an X-Ray Surveyor Mission, the STDT will organize multiple Science and Technology Workshops as well as conferences on the indisciplinary topics emphasizing synergies between different observational approaches and facilities.

\section{TECHNOLOGY CONSIDERATIONS}

The X-ray Surveyor science drivers indicate the need for complementary observational capabilities, which include fine imaging (e.g., for detecting the faintest sources), high resolution spectroscopy (e.g., to study diffuse gas in absorption, stellar atmospheres, central black hole engines), and spatially-resolved spectroscopy (e.g., to study the hot plasma in supernova remnants, galaxy halos and the circumgalactic medium, and clusters of galaxies). The suite of science instruments will be defined to implement these required capabilities.

\subsection{Configuration Trades}

The primary consideration in the mission design for X-ray Surveyor is to achieve great gains, particularly in sensitivity, over currently operating and proposed X-ray observatories, while maintaining high-angular resolution. The $X$-ray Surveyor concept utilizes revolutionary X-ray optics and cutting-edge instrumentation to reach these goals. To develop the mission concept, the STDT will first establish science requirements for angular resolution. The team will also look at optimizing the mirror optical scheme, taking into account considerations of the field of view size, the effective area, and the focal plane length. For example, longer focal lengths lead to improvements in high energy response but naturally limit the field-of-view size, while shorter focal lengths improve large survey capabilities. The team will also study the science drivers for the higher energy capabilities and look at various implementation options, including multi-layer mirror coatings.

\subsection{X-Ray Optics}

On-Going X-Ray Mirror Developments NASA currently funds multiple X-ray mirror development efforts that could potentially support an X-Ray Surveyor mission. A comprehensive summary of these efforts can be found in O'Dell et al, $2014^{3}$ and include segmented (glass and silicon, passive and active) and full-shell mirrors. 
To satisfy the requirements for an X-Ray Surveyor mission as described in the Astrophysics Roadmap, the angular resolution must be on the order of 1 " or better and one must be able to assemble large aperture areas ( $\sim 3 \mathrm{~m}$ or larger). In order to keep the total spacecraft mass down to make launch feasible, these optics should also be reasonably thin, or around $<2 \mathrm{~kg} / \mathrm{m}^{2}$ areal density ${ }^{3}$. The thinner the mirrors are, the more they are impacted by their environment. Considerations such as gravity sag, distortion due to thin-film stress and coatings, mounting-induced stress must be taken into account. Although these requirements may seem challenging, good progress is being made; and many of them are highlighted in these proceedings (Space Telescopes and Instrumentation 2016: Ultraviolet to Gamma-Ray, Conference 9905 and Advances in Optical and Mechanical Technologies for Telescopes and Instrumentation, Conference 9912.)

In addition to these NASA funded efforts, high-resolution, light-weight X-ray mirrors are also being developed in countries outside of the U.S. These developments include, but are not limited to, active X-ray mirrors in thin glass ${ }^{4}$, thin slumped glass foils ${ }^{5}$ and direct polishing of thin $\mathrm{SiO}_{2}$ substrates ${ }^{6}$. Multiple techniques to further improve mirror performance and reduce residual figure errors and mounting-induced stress are also being developed at various institutions around the globe, and include: differential deposition, ${ }^{7,8}$, ion-beam figuring ${ }^{9,10}$, low-stress coating techniques ${ }^{11}$, differential ion implantation ${ }^{12}$, active figure correction ${ }^{13}$ and magnetostrictive corrections ${ }^{14}$. The citations noted are representative, as there are multiple groups working on each of these efforts.

The X-Ray Surveyor STDT combined with the OWG has been tasked with developing a Technology Roadmap that would define the path for achieving an optical assembly that will be complimentary to the science requirements. This group will consider multiple technology development paths that may be capable of achieving a technology maturity level consistent with NASA's mission study requirements. To facilitate an affordable solution to large-scale, high-precision, high-repeatability fabrication, this group will also consider industry capabilities and leveraging resources from other government laboratories. The X-ray Surveyor Study Office is tasked by the STDT to perform systems engineering studies and develop tools to help understand the performance of various X-ray optics technologies. The goal is to ensure the development of at least one telescope point-design that meets the Science Requirements determined by the STDT. This point-design will be used in the $X$-ray Surveyor mission configuration studies.

\subsection{Potential Science Instruments}

$X$-ray Surveyor's unique science is enabled primarily by $\mathrm{X}$-ray mirrors with superior angular resolution and large collecting area. Several notional science instruments that can best exploit the new telescope's properties are in discussion. These instruments will provide further gains in sensitivity as well as spectroscopic capabilities to the $X$-ray Surveyor, enabling science which is very different from Athena, and will put X-ray Surveyor firmly into the array of great space- and ground-based astronomical facilities expected to arrive by late 2020 's.

The strawman $X$-ray Surveyor instruments are cutting edge and are currently under development by several research groups. The X-Ray Imaging Microcalorimeter Spectrometer (XMIS) has a $5^{\prime} \times 5^{\prime}$ field-of-view filled with $1^{\prime \prime}$ pixels covering the energy range from 0.2 to $10 \mathrm{keV}$ and will provide better than $5 \mathrm{eV}$ resolution. The High Definition X-Ray Imager (HDXI) is a CMOS active pixel sensor with $0.33^{\prime \prime}$ pixels covering a $22^{\prime} \times 22^{\prime}$ field of view and sensitive to X-rays in the energy range from $0.2-10 \mathrm{keV}$. HDXI chips approximately follow the optimal focal surface of the X-ray mirror in the soft band. The Critical Angle Transmission (CAT) gratings may provide high-efficiency spectroscopy with resolving power of $\sim 10,000$ in the $0.2-1.2 \mathrm{keV}$ band $^{15}$. An alternative grating spectrometer utilizing off-plane reflection gratings is also an option which will be assessed in future mission studies ${ }^{16}$. At present, this is a strawman design, where future iterations will trade competing technologies for the telescope and the science instruments.

\subsection{Technology Roadmap}

Understanding the cost and feasibility of the mission concept depends on the fidelity of the spacecraft design, the optics, and the science instruments. In the case of the X-Ray Surveyor, the main contributors are the X-ray mirrors (and full assembly) and the science instruments. A Technology Roadmap will be developed that includes the mirrors as well as each science instrument. This Roadmap will assess and compare existing efforts in order to define feasible paths forward and will highlight opportunities for breakthrough innovations. In this case, feasible means within a reasonable cost and timeframe for development to the required NASA technology maturity level, consistent with a Decadal mission. 
The Roadmap will define the state-of-the-art for each technology, assess the key parameters related to defining the observatory, and define the development schedule for each technology along with associated costs for each year of the development. Each technology will be broken down into its constituent parts and assessed. For example, the mirror assembly may be broken down into the substrate, coating process, alignment and bonding, and module design, analysis and testing. Multiple development paths for each technology will be considered.

In 2014, NASA assembled a Technology Roadmap development team that used a systematic process to create roadmaps for multiple mission classes ${ }^{17}$. The process is illustrated in Figure 1, and summarizes an example path for assembling a Roadmap. This particular path requires defining a Mission Class and relating it to potential technologies, to stakeholder needs, and to NASA priorities.

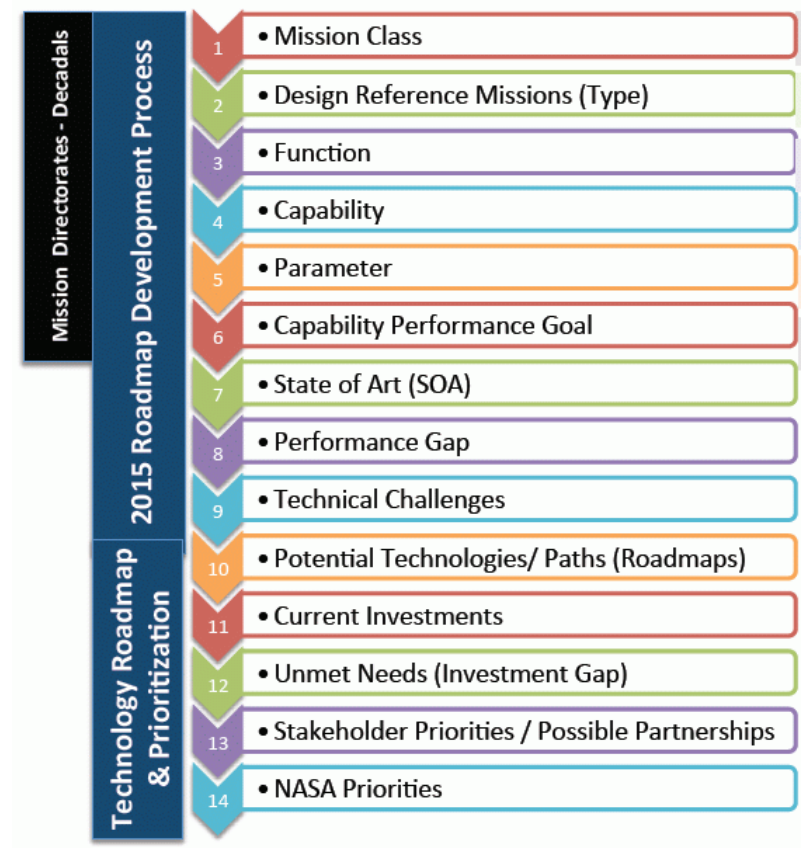

Figure 1. Flow diagram of the NASA Technology Roadmap process. This is one of many processes that the STDT and WGs may use to compile the Roadmap.

The priorities of the broad community are of utmost importance to the STDT. The study team and OWG will solicit community feedback throughout the development of the Roadmap to ensure that all resources are being considered, and that the most efficient path(s) forward are defined.

\section{SUMMARY}

The X-Ray Surveyor is the next generation large X-ray observatory. Much like the Chandra X-Ray Observatory, the $X$ Ray Surveyor will provide breakthrough X-ray science while complementing the many observatories operating at other wavelengths.

The STDT is currently in the process of defining key science drivers for an X-Ray Surveyor mission. These drivers will dictate the observatory requirements that will then determination the mission concept, top-level schedule and cost estimates. The SWGs, OWG and IWG are currently accepting new members. The WGs are an essential part of the study as they allow for the community to have a voice in the purpose and design of such a mission. The international community and industry are encouraged to participate. 
All of the STDT meetings and teleconferences are open to the public, and information is regularly updated on the X-Ray Surveyor website (http://wwwastro.msfc.nasa.gov/xrs/).

We hope that you will join us in defining the next Great Observatory.

\section{ACKNOWLEDGEMENTS}

The authors would like to thank the Astronomy Community and NASA for their support for the X-Ray Surveyor Mission. It is important that the $X$-Ray Surveyor is designed to be relevant and useful to the Astronomy Community as a whole. We welcome your input and will strive to achieve an instrument that will be beneficial to all.

\section{REFERENCES}

${ }^{1} \mathrm{http} / / / \mathrm{www} . n a p . e d u / c a t a l o g / 12951 /$ new-worlds-new-horizons-in-astronomy-and-astrophysics

${ }^{2} \mathrm{http}: / /$ science.nasa.gov/science-committee/subcommittees/nac-astrophysics-subcommittee/astrophysics-roadmap/

${ }^{3}$ O'Dell, Stephen L., et al. "Toward large-area sub-arcsecond x-ray telescopes." SPIE Optical Engineering+ Applications. International Society for Optics and Photonics, 2014.

${ }^{4}$ Spiga, D., et al. "Manufacturing an active X-ray mirror prototype in thin glass." Journal of synchrotron radiation 23.1 (2016).

${ }^{5}$ Basso, Stefano, Marta Civitani, and Giovanni Pareschi. "Design of a medium size x-ray mirror module based on thin glass foils." SPIE Astronomical Telescopes+ Instrumentation. International Society for Optics and Photonics, 2016.

${ }^{6}$ Pareschi, G., et al. "Beyond Chandra: problems, solutions, and perspectives for the implementation of very high angular resolution x-ray telescopes in the new millennium." SPIE Astronomical Telescopes + Instrumentation. International Society for Optics and Photonics, abstract \# 9905-61, 2016.

${ }^{7}$ Windt, David L., and Ray Conley. "Two-dimensional differential deposition for figure correction of thin-shell mirror substrates for x-ray astronomy." SPIE Optical Engineering + Applications. International Society for Optics and Photonics, 2015.

${ }^{8}$ Kilaru, Kiranmayee, et al. "Progress in differential deposition for improving the figures of full-shell astronomical grazing incidence x-ray optics." SPIE Optical Engineering + Applications. International Society for Optics and Photonics, 2015.

${ }^{9}$ Yamada, Jumpei, et al. "Development of ion beam figuring system with electrostatic deflection for ultraprecise X-ray reflective optics." Review of Scientific Instruments 86.9 (2015): 093103.

${ }^{10}$ Civitani, M., et al. "Ion beam figuring of thin glass plates: achievements and perspectives." SPIE Astronomical Telescopes+ Instrumentation. International Society for Optics and Photonics, 2016.

${ }^{11}$ Chan, Kai-Wing, et al. "Coating thin mirror segments for lightweight x-ray optics." SPIE Optical Engineering+ Applications. International Society for Optics and Photonics, 2013.

${ }^{12}$ Chalifoux, Brandon, et al. "Gas bearing slumping and figure correction of x-ray telescope mirror substrates." SPIE

Astronomical Telescopes + Instrumentation. International Society for Optics and Photonics, 2016.

${ }^{13}$ Reid, Paul B., et al. "Development Status of Adjustable X-ray Optics with 0.5 Arcsec Imaging for the X-ray Surveyor Mission Concept." AAS/High Energy Astrophysics Division. Vol. 15. 2016.

${ }^{14}$ Wang, Xiaoli, et al. "Investigation of magnetically smart films applied to correct the surface profile of light weight Xray optics in two directions." SPIE Optical Engineering + Applications. International Society for Optics and Photonics, 2015.

${ }^{15}$ Heilmann, Ralf K., et al. "Critical-angle x-ray transmission grating spectrometer with extended bandpass and resolving power> 10,000." SPIE Astronomical Telescopes+ Instrumentation. International Society for Optics and Photonics, 2016.

${ }^{16}$ McEntaffer, Randy L., "Advancements in x-ray reflection gratings for space-based applications." SPIE Astronomical

Telescopes+ Instrumentation. International Society for Optics and Photonics, abstract \# 9905-64, 2016.

${ }^{17}$ http://www.nasa.gov/offices/oct/home/roadmaps/index.html 